\title{
FREAK WAVE AND WEATHER CONDITION
}

\author{
Nobuhito Mori ${ }^{1}$, Hajime Mase $^{1}$ and Tomohiro Yasuda ${ }^{1}$
}

\begin{abstract}
The kurtosis of the surface elevation, Benjamin-Feir Index (BFI) and directional spread are measures of nonlinear four-wave interactions and freak waves. The dependence of kurtosis, BFI and directional spread under typhoon conditions are examined by numerical simulations. The BFI is significantly large in the fourth quadrant of the typhoon while the directional spread is small in the fourth quadrant. It was found that the potentially possible area of freak wave occurrence is the fourth quadrant of the typhoon rather than the other quadrants.

Keywords: freak wave, extreme wave, typhoon, BFI, directional spread
\end{abstract}

\section{INTRODUCTION}

Freak waves are sometimes featured by a single and steep crest causing severe damage to offshore structures and ships. The last decade freak waves have become an important topic in engineering and science. Freak wave studies started in the late 80's (Dean, 1990) and high-order nonlinear effects on the freak waves were discussed in the early 90's at several engineering conferences (Yasuda et al., 1992; Yasuda and Mori, 1994). Evidence of freak wave generation in the real ocean was reported from field observations of the North Sea (Stansell et al., 2003; Guedes Soares et al., 2003), the Sea of Japan (Yasuda and Mori, 1997; Yasuda et al., 1997) and the Gulf of Mexico (Guedes Soares et al., 2004). Due to the many research efforts, the occurrence of freak waves, their mechanism and detailed dynamic properties are now becoming clear (Trulsen and Dysthe, 1997; Lavrenov, 1998; Osborne et al., 2000; Onorato et al., 2001; Mori et al., 2002). The state of the art on freak waves was well summarized at the last three Rogue Wave Conferences, held in 2000, 2004 and 2008, and a review by Dysthe et al. (2008).

Freak wave generation is sometimes discussed in the context of the nonlinear instability in deep-water waves because of the similarity of the steep wave profile itself (Yasuda et al., 1992; Onorato et al., 2001). Janssen (2003) found that the ratio between the steepness, a measure of the nonlinearity and the spectral band-width, a measure of the dispersion, is an important parameter for determining the probability of finding a large wave. After Janssen (2003) this ratio between nonlinearity and dispersion was named the Benjamin-Feir-Index, BFI; its relation to the kurtosis has been found in Janssen (2003) in the limit of large times and for narrow banded spectra neglecting directional dispersion. Define the Benjamin-Feir-Index as

$$
B F I=\frac{\sqrt{2} \epsilon}{\delta_{\omega}}
$$

where $\epsilon$ and $\delta_{\omega}$ are the characteristic wave steepness and the typical bandwidth of frequency spectrum, respectively. Then, for narrow-band,unidirectional waves, the kurtosis $\mu_{4}$ becomes

$$
\mu_{4}-3=\kappa_{40}=\frac{\pi}{\sqrt{3}} B F I^{2}
$$

where $\kappa_{40}$ is the fourth order cumulant of the surface elevation probability distribution which is equal to kurtosis minus three $\left(\kappa_{40}=\mu_{4}-3\right)$. The final result is that the kurtosis depends on the square of the BFI. The role of the skewness in the wave height distribution is less important with respect to kurtosis. The skewness comes usually as a result of second order corrections (bound modes) and is weakly affected by the dynamics of free waves (Onorato et al., 2005). In

${ }^{1}$ Disaster Prevention Research Institute, Kyoto University, Gokasho, Uji, Kyoto 611-0011, JAPAN. 


\section{COASTAL ENGINEERING 2010}

Mori and Janssen (2006) the formal relation between kurtosis, the maximum wave height and occurrence probability of freak wave has been discussed. The kurtosis enters in the maximum wave height distribution function as a nonlinear correction to the Rayleigh distribution; when the kurtosis tends to three, the expected Gaussian values, then the distribution function tends to the Rayleigh distribution. In this context, the changes in the kurtosis can be evaluated once the evolution of the wave spectrum is known (see Janssen, 2003). In Mori and Janssen (2006) the wave height exceedance probability is also derived to formulate occurrence probabilities of freak wave as a function of the considered number of waves. This theoretical framework of freak wave prediction was verified by large scale wave experiments for unidirectional waves and predicts well the spatial developments of the kurtosis, the wave height distribution and the maximum wave height distribution in the wave tank (Mori et al., 2007) and field observation (Petrova and Guedes Soares, 2008). This is of course not a surprise because the kurtosis is a fourth-order moment of a probability density function (pdf), therefore it is a measure of the importance of the tails in a distribution. Moreover, the fourth-order moment of surface elevation is related to a third-order nonlinear interactions (Longuet-Higgins, 1963) providing consistency between the statistical and dynamical point of views. Thus, an accurate estimation of kurtosis is of paramount interest for freak wave prediction.

In addition, recent works based on numerical simulations of envelope equations and wave tank experiments have pointed out the influence of directional dispersion on the kurtosis evolution in deep-water. Gramstad and Trulsen (2007) showed a decrease of kurtosis and maximum wave height for directional waves in comparison with unidirectional waves based on a series of the modified NLS. Waseda (2006) and Onorato et al. (2009) investigated the monotonic decrease of kurtosis as directional effects become significant. These results show that the directional dispersion effects suppresses the kurtosis enhancement in directional sea states. Therefore, a more general expression for the kurtosis in directional sea states is required. For example, Mori et al. (2010) proposed semiempirical relation of reduction of kurtosis due to directional effects introducing directional spread $\sigma_{\theta}=\int \theta^{2} D(\theta) d \theta$ from directional function $D(\theta)$;

$$
\begin{gathered}
\kappa_{40}=\frac{\pi}{\sqrt{3}} \times B F I_{e f f}^{2} \\
B F I_{\text {eff }}^{2}=\frac{B F I^{2}}{1+\alpha R}
\end{gathered}
$$

where $B F I_{\text {eff }}$ is effective BFI including directional dispersion effects, $R=\delta_{\theta}^{2} / 2 \delta_{\omega}^{2}$ is ratio between frequency and directional dispersions, and $\alpha$ is turning coefficient respectively. This kind of directional dispersion effects were verified by several authors (i.e. Onorato et al., 2009). The discussion about freak wave prediction and parameterization have been carried out by the steady condition such as mechanically generated wave flumes and potential wave theory. Therefore, it is required to know the behavior of freak wave parameters in unsteady weather condition.

Following above mentioned summary, the freak wave occurrence is significantly large for the case of steep wave slope, narrow directional bandwidth and narrow frequency bandwidth waves. The purpose of the present paper is to examine the possible condition of freak wave in the typhoon condition based on a series of numerical simulations by spectral wave model. 


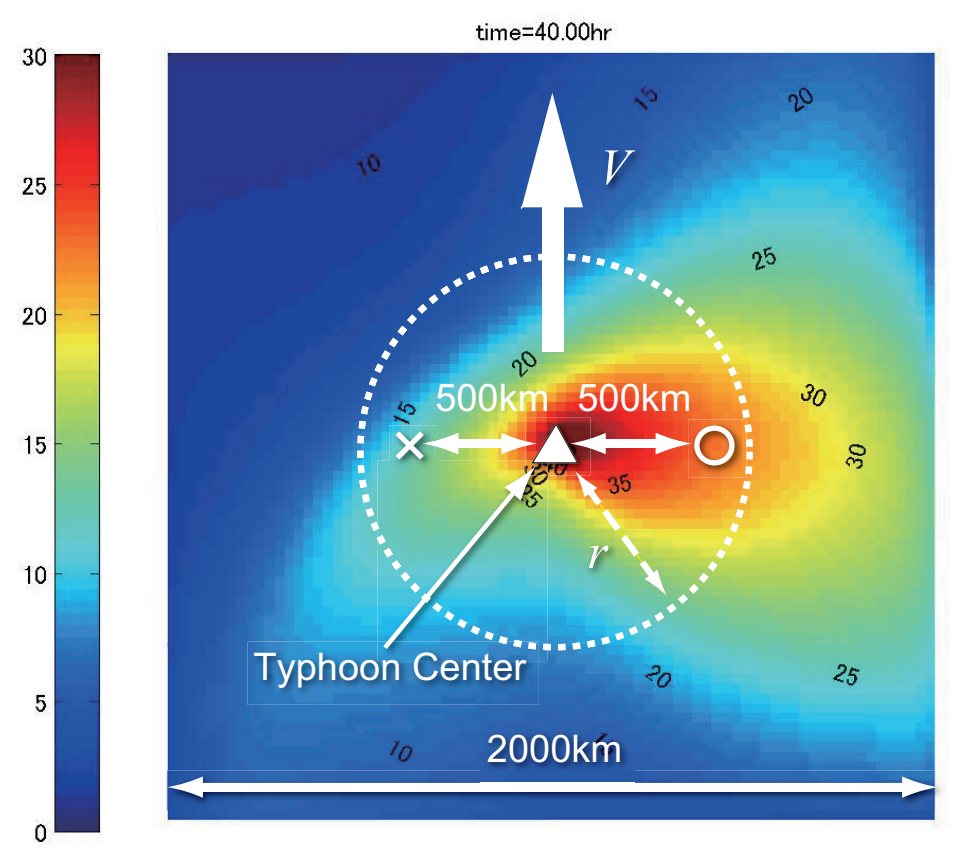

Figure 1. Illustration of parameters of empirical typhoon model ( $V$ : typhoon velocity, $r$ : typhoon radius), numerical domain and specific locations for analysis

\section{NUMERICAL METHOD}

The numerical experiments were conducted by a spectral wave model, SWAN model (Booij et al., 1999), giving wind fields by an empirical typhoon model so-called super gradient wind by Fujii and Mitsuta (1986) (denotes SGW hereafter for simplicity). The SGW was used to check the sensitivity of typhoon condition to extreme wave characteristics under the controlled realistic unsteady conditions. Keeping a constant central pressure but changing characteristic radius and moving speed of typhoon, it is possible to understand freak wave related parameters in the unsteady weather condition. The analysis of both temporal and spatial wave growth and decay, the characteristics of dangerous condition of freak wave occurrence can be found.

The spectral wave model was configured as a quasi-linear theory for wave energy input, $S_{i n}$, and Komen's scheme for wave energy dissipation, $S_{d i s}$, but was selected two different nonlinear transfer functions, $S_{n l}$, of DIA and MDIA to check the sensitivity of nonlinear interactions to the wave field. Over all the sensitivity of $S_{n l}$ to the target results was not significant but it will be discussed in the next section. The numerical simulations were performed in the rectangular domain of $2000 \mathrm{~km}$ by $2000 \mathrm{~km}$ with $5 \mathrm{~km}$ resolution. The discretization of directional spectra was 40 bins in frequency space and 36 bins in directional space, respectively.

The wind field parameters given by the SGW with fixed central pressure as $-60 \mathrm{hPa}$ from environment atmosphere were changed typhoon moving speed $V$ from $30 \mathrm{~km} / \mathrm{h}$ to $50 \mathrm{~km} / \mathrm{h}$ and characteristic wind radius $r$ from $50 \mathrm{~km}$ to $100 \mathrm{~km}$, respectively. The illustration of parameters $V$ and $r$ of typhoon model is shown in Fig.1. The typhoon moves from the south to north which 


\section{COASTAL ENGINEERING 2010}

corresponds bottom to top in Fig.1. The three marks (open circle, triangle and closed circle) in the figure indicate specific locations for the analysis of temporal change of wave characteristics in next section. These three locations were selected as north and south cross section of typhoon path, one is typhoon center, and the others are $\pm 500 \mathrm{~km}$ in east and west direction from the typhoon center as shown in Fig.1.

The results of numerical experiments were analyzed to focus on 1) spatial distributions of freak wave parameters around the typhoon eye, 2) temporal changes of freak wave parameters at different locations in respect to the relative position to typhoon eye. The numerical condition is not realistic but it can be understand general behavior of freak wave parameters and dangerous condition of freak wave occurrence.

\section{RESULTS AND DISCUSSION}

The typhoon wind and wave fields given by the SGW were propagated from the south to north end of the computational domain (see Fig.1). At the time of typhoon eye passes at the center of computational domain, it is 40-70 hours after initial condition. Then the wave field can be regarded as quasi-stationary condition because of the enough time and space of wave growth. Therefore, it is useful to analyze the snapshot of spatial distribution of freak wave related parameters around the typhoon center at quasi-stationary time. The time and space can be exchanged each other due to constant typhoon speed.

Fig.2 shows both spatial distributions of freak wave parameters and time series of directional spectra at two locations. The vertical panels in the middle of Fig.2 show spatial distributions of BFI, $H_{s}$ and $\sigma_{\theta}$ around typhoon eye in the case of $V=50 \mathrm{~km} / \mathrm{h}, r=50 \mathrm{~km}$, respectively. The typhoon eye is located at the center of figure. The typhoon moves from the south to north (bottom to top in the figure) with constant speed of $V$. The significant wave height $H_{s}$ distribution indicates axis asymmetric at the center (north-south) line and the value of $H_{s}$ in the east side is larger than that of west side. This is due to asymmetric profile of given wind field by the SGW. The wind profile difference is due to the asymmetry of pressure field and additional effects of typhoon moving speed in both sides. The east side area is added additional wind speed and west side area is subtracted. The maximum value of $H_{s}$ is located at the center of typhoon and it is rapidly decreased in the west side, although large $H_{s}$ is continued from typhoon eye in the east side. Eq.(1)-Eq.(4) indicate the possible dangerous condition of freak wave is the wave field which has narrow directional spectra, in other words, the wave field should has large BFI and small directional spread. Remind these parameter combination, the numerical experiments of typhoon wave give impressive spatial distributions both BFI and directional spread $\sigma_{\theta}$, respectively. The spatial distribution of BFI is large in the fourth quadrant in respect to typhoon eye. There is a ridge of large BFI from the typhoon eye to the south east direction in the fourth quadrant and the value exceeds more than 0.6 at that area. However, BFI in the third quadrant (the south west) is always smaller than 0.3. It means that the freak wave occurrence follows linear Rayleigh wave theory in the third quadrant under the given condition (Mori and Janssen, 2006). The wave steepness is large in the fourth quadrant and therefore the value of BFI becomes significantly large in this area. The other hand, the spatial distribution of directional spread around typhoon is also different $H_{s}$ and BFI. The directional spread is large in the behind (south) of the typhoon center and some area in the west of typhoon eye. Previous studies (Onorato et al., 2009) indicate that the large directional spread cancels the nonlinear enhancement of freak wave occurrence which represents by BFI, Eq.(1). Thus, the wave field in the third quadrant follows linear theory of prediction of freak wave. Following these results, the wave field in the fourth quadrant of typhoon (southeast from typhoon eye) has larger BFI and narrower directional spread, that is, the possible freak wave occurrence area under the typhoon condition.

The temporal changes of directional spectra along the typhoon eye and east side are shown in 

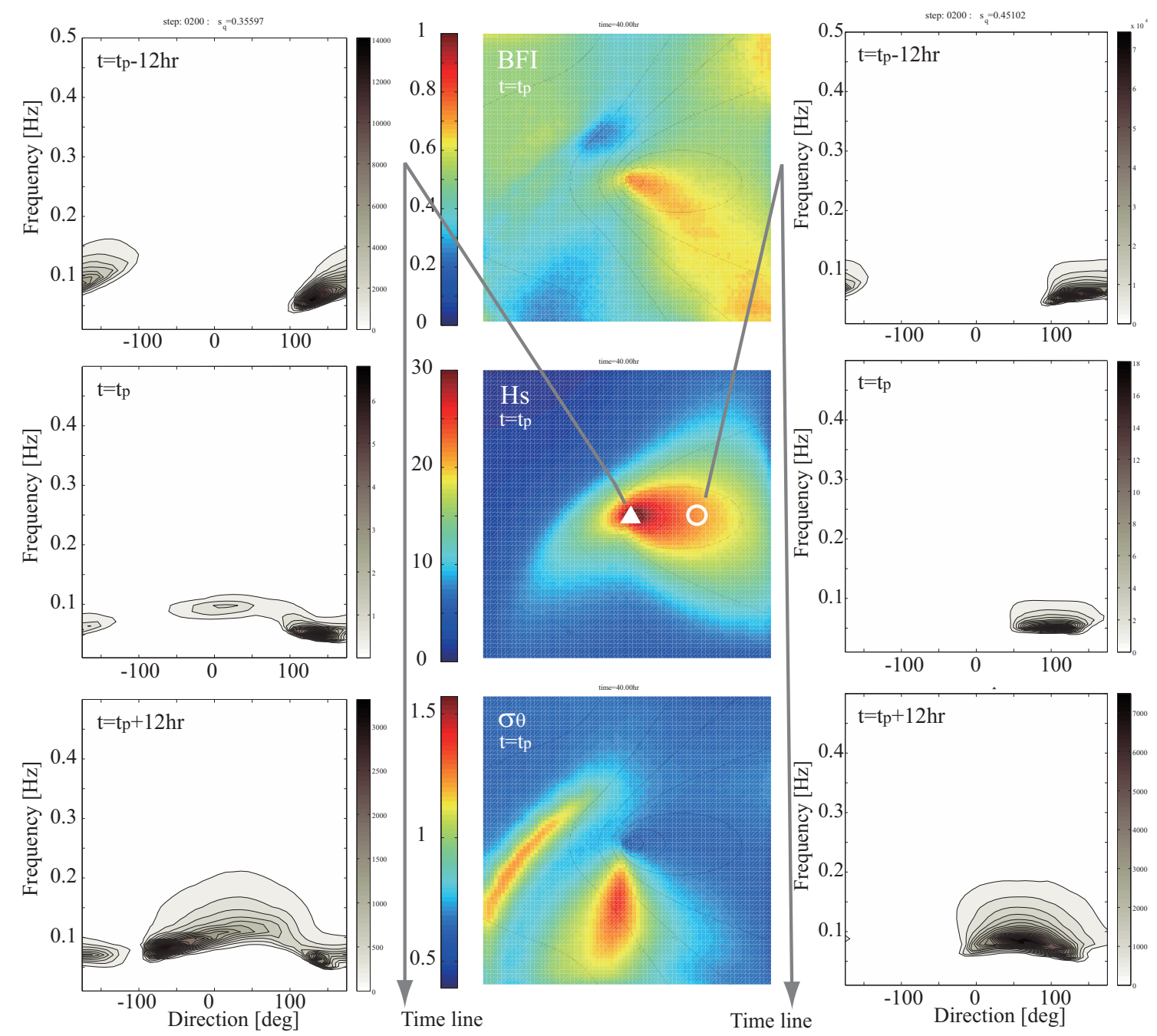

Figure 2. Spatial distribution of BFI, $H_{s}$ and $\sigma_{\theta}$ around typhoon eye (is located at the center of figure) and temporal change of directional spectra (left: typhoon center, right: $+500 \mathrm{~km}$ east from typhoon center, time line starts from top to bottom) ( $V=50 \mathrm{~km} / \mathbf{h}, r=50 \mathbf{k m})$

Fig.2. The single peak directional spectra can be observed in the east side but two peak directional spectra can be seen after closest approach of typhoon in the center section. This is reason why the directional spread becomes large in the south of typhoon. However, the bimodal sea states is also dangerous generating crossing/chopping waves, although the nonlinear enhancement of freak wave occurrence is weak in bimodal sea states. The qualitative characteristics of directional spectra around the typhoon eye is agreed with field observation of hurricane waves by Young (2006).

The spectral wave model gives only wave energy information but freak wave phenomena itself is individual one or a few extreme waves in the random wave train. Using the nonlinear statistical theory predicting the maximum wave height from the wave spectra by Mori et al. (2010), the 


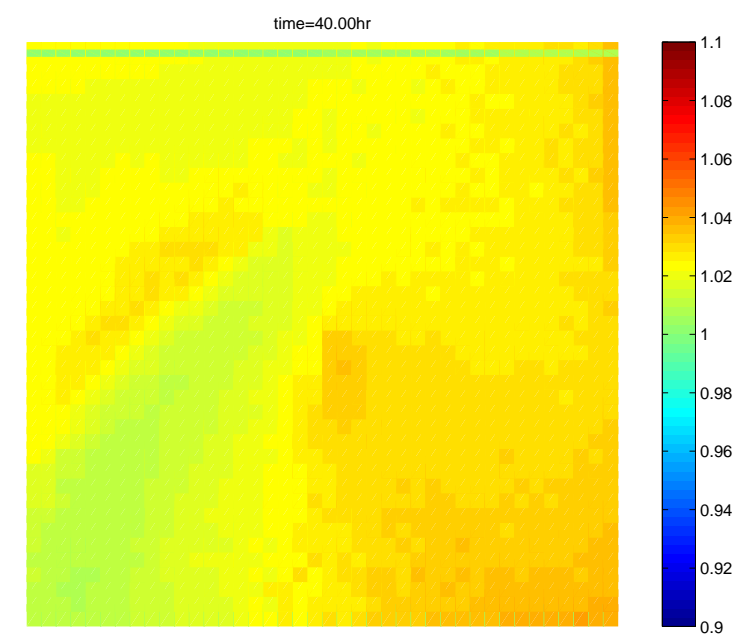

Figure 3. Spatial distribution of estimated $H_{\max }$ normalized by linear wave theory $(V=50 \mathbf{k m} / \mathbf{h}, r=50 \mathbf{k m})$

ratio of nonlinear enhancement of maximum wave height to linear Rayleigh wave theory can be estimated.

$$
R\left(H_{\text {max }}\right)=\frac{\int p_{\text {nonlinear }}\left(B F I, \sigma_{\theta}, T_{\text {storm }}\right) d H_{\text {max }}}{\int p_{\text {linear }}\left(T_{\text {storm }}\right) d H_{\max }}
$$

where $p_{\text {nonlinear }}$ is the probability density function (pdf) of maximum wave height by the nonlinear theory as functions of BFI, $\sigma_{\theta}$ and storm duration $T_{\text {storm }}, p_{\text {linear }}$ is the pdf of maximum wave by the linear theory as a function of the storm duration. The ratio $R\left(H_{\max }\right)$ means expected ratio of maximum wave height enhancement in comparison with the linear theory. Assuming the storm duration $T_{\text {storm }}=30$ min, Fig.3 shows spatial distribution of nonlinear enhancement of $H_{\max }$ normalized by the linear theory. The typhoon eye is located at the center of figure similar to previous figure. The nonlinear enhancement of maximum wave in the fourth quadrant is $3-5 \%$ larger than surround area, although it is almost linear $(=1)$ in the third quadrant. Therefore, the wave field in the fourth quadrant of typhoon (southeast from typhoon eye) is much dangerous for freak wave occurrence than the other quadrants.

The previous discussion was conducted by the one particular typhoon condition of $V=50$ $\mathrm{km} / \mathrm{h}$ and $r=50 \mathrm{~km}$. The sensitivity of typhoon velocity and radius were performed changing $V$ and $r$, respectively. There is no significant difference of typhoon radius but typhoon velocity speed shows different characteristics of freak wave parameters. Fig.4 shows spatial distribution of BFI for different speeds of typhoon in the case of $r=50 \mathrm{~km}$. The slower typhoon moving speed gives concentrated distribution of freak wave parameters around the typhoon eye but longer typhoon speed gives more asymmetric BFI distribution. The superimpose of gradient wind and moving speed gives different solution and faster typhoon $(V=50 \mathrm{~km} / \mathrm{h})$ has longer tail of BFI toward the southeast direction from the eye. The spatial distribution of $H_{s}$ as shown Fig.2 (middle panel) indicates $H_{s}$ peak appeared east and west direction but the BFI peak appeared after that in the fourth quadrant. Although, the value of $H_{s}$ in the east side is slightly smaller than the peak of storm, but potential possibility of freak wave is much longer after the peak of $H_{s}$ appeared in the fourth quadrant. This is unexpected interesting result and should be discussed the later part of 


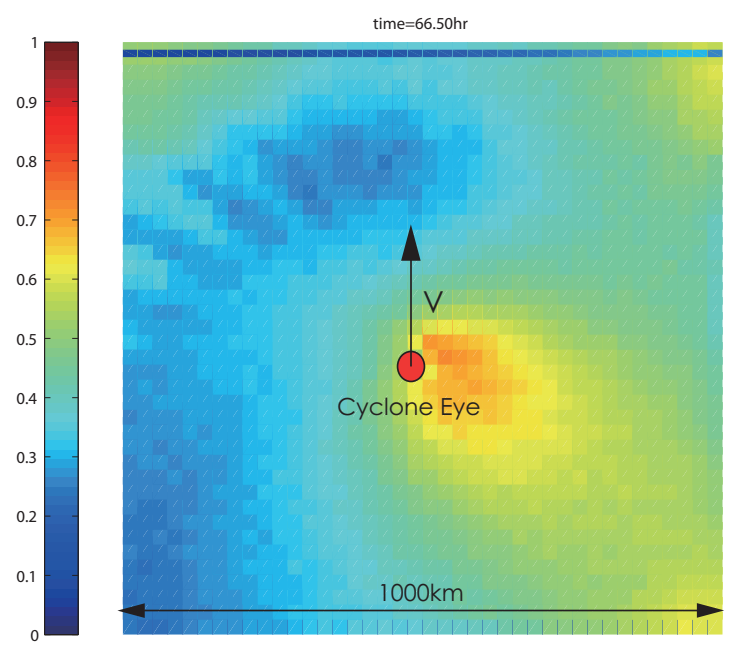

(a) $V=30 \mathrm{~km} / \mathrm{h}$

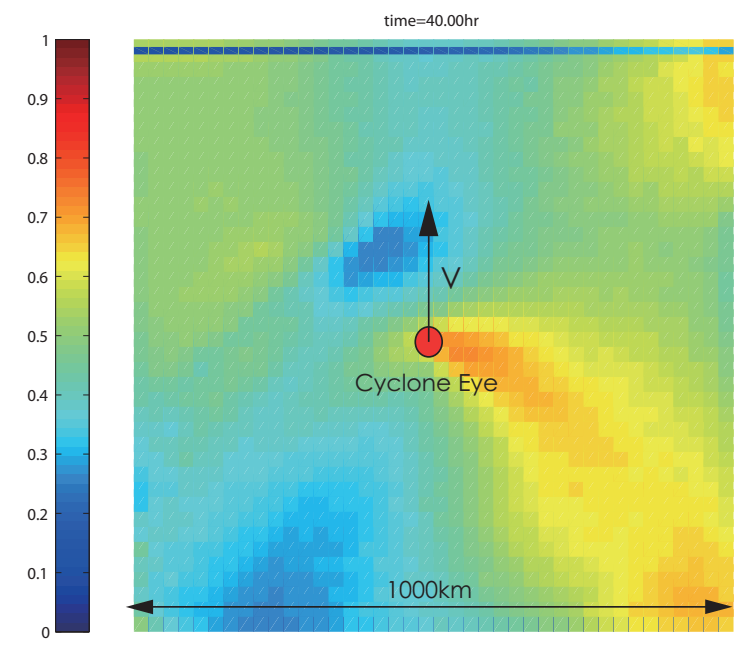

(b) $V=50 \mathrm{~km} / \mathrm{h}$

Figure 4. Dependence of spatial distribution of BFI on typhoon speed $(r=50 \mathrm{~km})$

this section.

The time series of freak wave parameters at different locations are important to understand qualitative behavior and are easy to compare with different conditions. Fig.5 shows time series of freak wave related parameters at three different locations ( $\circ: 500 \mathrm{~km}$ west, triangle: typhoon center, - $500 \mathrm{~km}$ east) in the case of $V=50 \mathrm{~km} / \mathrm{h}$ and $r=50,100 \mathrm{~km}$ ), respectively. The differences of typhoon radii are generally difference of temporal duration with similar time series. Therefore, we will focus on the differences of locations. The time history of $H_{s}$ along the north south section of typhoon center indicates rapid growth until $t=40 \mathrm{hr}$ (closest approach of typhoon) and decay near the typhoon eye and relatively mild change both sides of area. Obviously, the maximum value of $H_{s}$ in the west side is smaller than the others as we expected. The values of BFI shown in Fig.5 (b) were started the same values initially (with very small $H_{s}$ ) and show different behavior after that. Similar to $H_{s}$, BFI at the typhoon center line in the north-south section shows rapid growth 


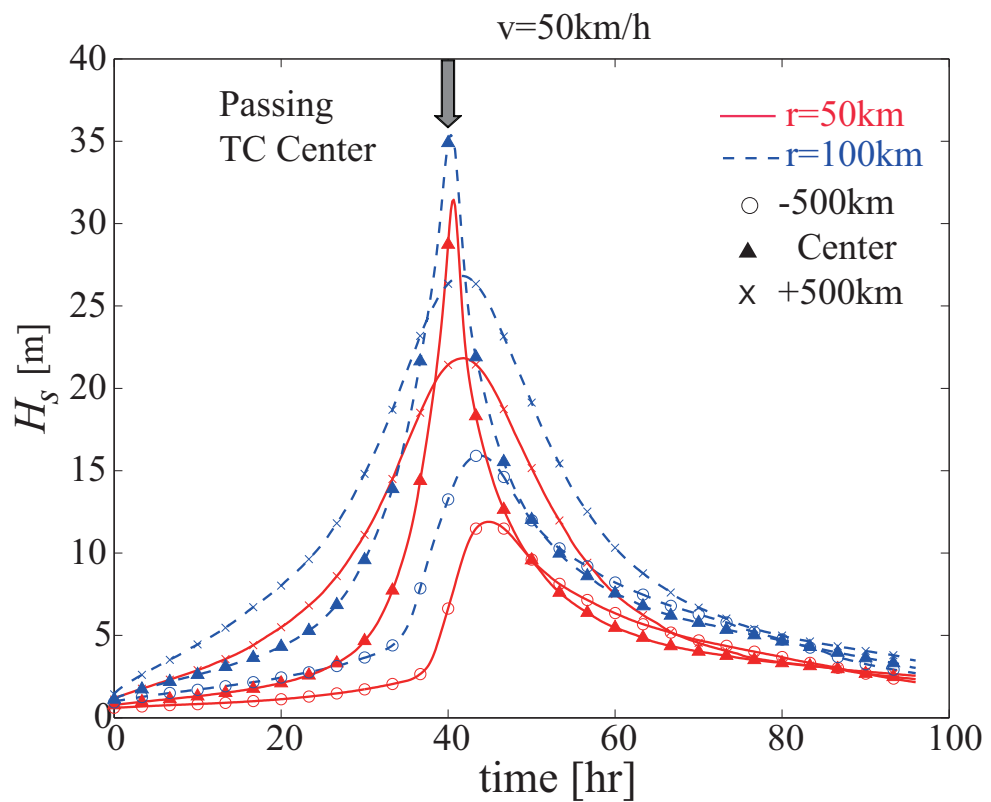

(a) $H_{s}$

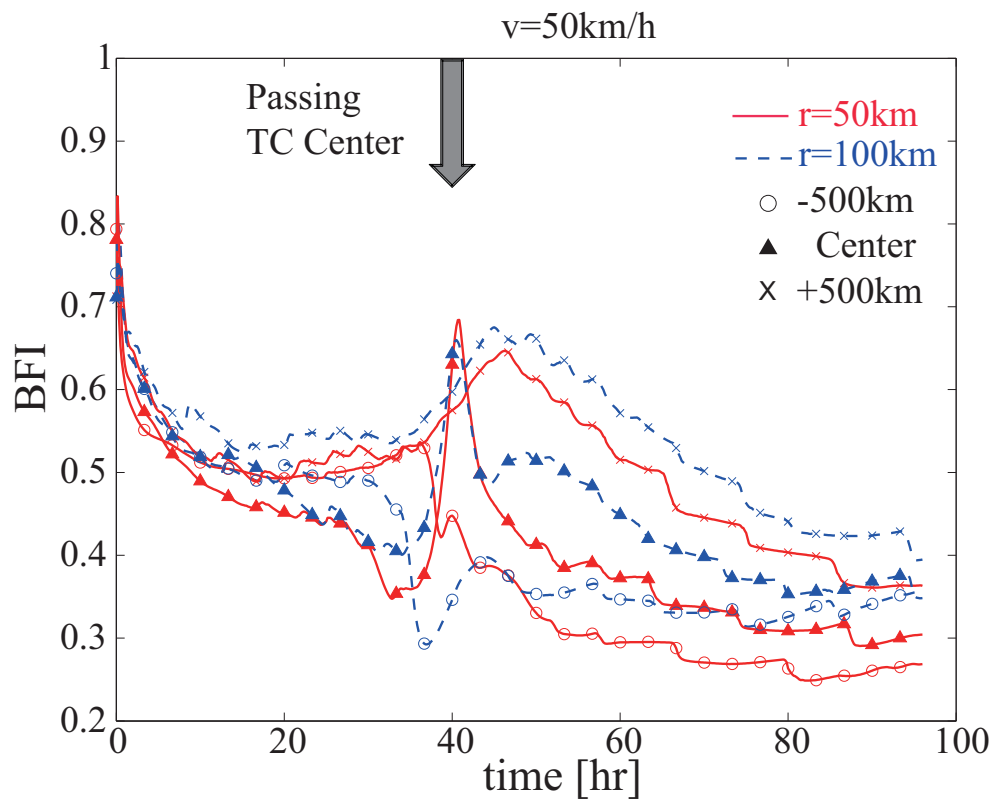

(b) BFI

Figure 5. Time series of freak wave related parameters at three different locations ( $\circ: 500 \mathbf{~ k m}$ west, triangle: typhoon center, $\bullet$ : $\mathbf{5 0 0} \mathbf{~ k m}$ east; $V=50$ $\mathbf{k m} / \mathbf{h}, r=50,100 \mathbf{~ k m}$ ) 


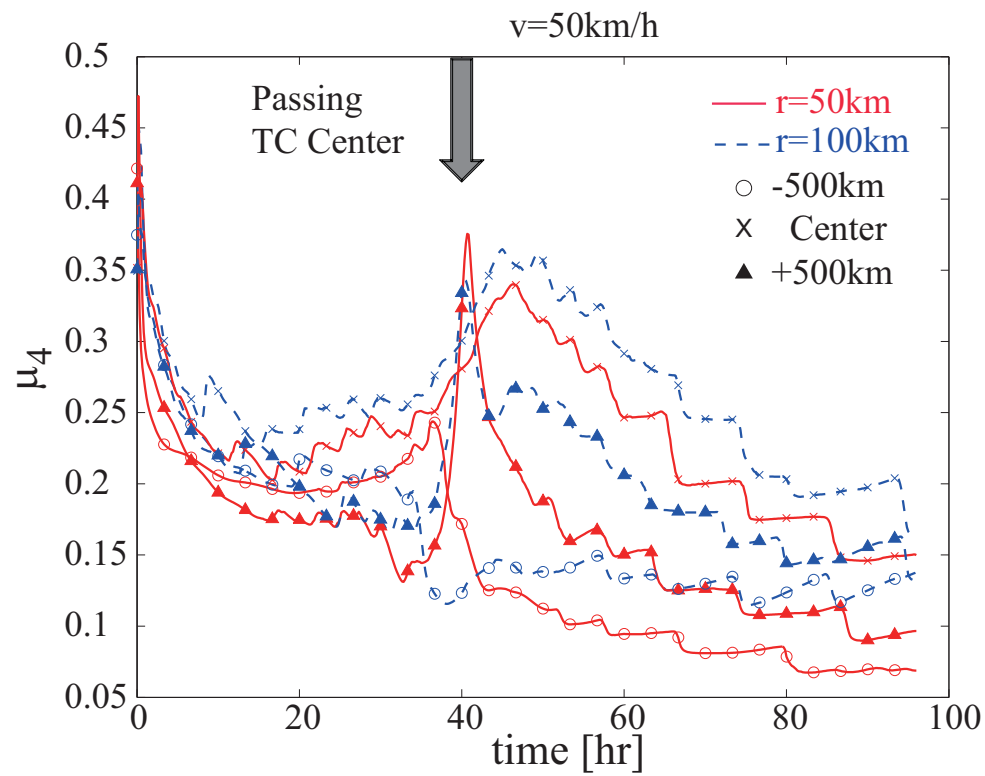

(c) kurtosis minus three

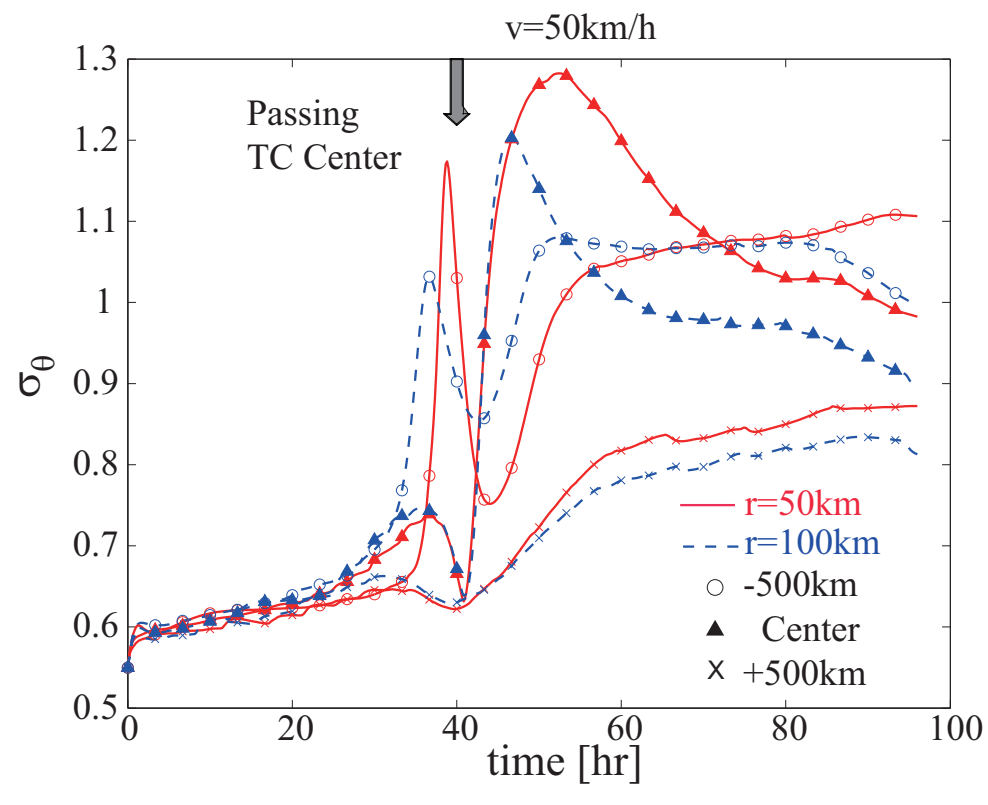

(d) $\sigma_{\theta}$

Figure 5. Time series of freak wave related parameters at three different locations ( $\circ: 500 \mathbf{~ k m}$ west, triangle: typhoon center, $\bullet: 500 \mathbf{~ k m}$ east; $V=50$ $\mathbf{k m} / \mathbf{h}, r=50,100 \mathbf{~ k m})$ (cont.) 


\section{COASTAL ENGINEERING 2010}

and decay but which mechanism is different. The BFI is defined by wave steepness divided by wave frequency bandwidth. The frequency bandwidth becomes broader near the peak of storm and becomes boarder after typhoon eye passed in the east side of typhoon. And then, the value of BFI is reached at the maximum a few hours after the $H_{s}$ reached at maximum in the east side of typhoon and it continued more than half or one day after typhoon passed. The is main mechanism of BFI grow-up after peak of typhoon. Therefore, the potentially dangerous situation of freak wave occurrence keeps longer in the east side of typhoon. However, the value of BFI in the west side is dropped down and it always below the negligible level $(\mathrm{BFI}<0.4)$. Additionally, the behavior of kurtosis defined by Eq.(2) shows the same time series but it implies physically meaningful information. The kurtosis is a measure of deviation from the normal distribution and is 3 if waves obey the normal distribution. Mori et al. (2007) and the others investigated that the nonlinear enhancement becomes significant if kurtosis exceeds more than 3.3-3.5. Such large value of kurtosis can be only observed at the typhoon center in very short-time and it continued a while after closest approach of typhoon in the east side. Thus, the nonlinear enhancement of freak wave is only significant in the east side of typhoon after closest approach which is equivalent discussion in previous paragraph. Finally, Fig.5 (d) shows the time series of directional spread which suppress the nonlinear enhancement of freak wave occurrence. The directional spread, $\sigma_{\theta}$, is small initially but is increased after closest typhoon approach. The value of $\sigma_{\theta}$ is fluctuated violently at the center and is rapidly increased after the closest approach due to bimodal spectra as shown in Fig.2. However, the directional spread keeps relatively smaller value $(<0.8)$ in the east side location. In other words, the fourth quadrant can be regarded as quasi-steady states such as the experimental wave flume. However, the third quadrant, specially the south area of typhoon eye, is the unsteady, bimodal and rapid change of wind and wave directions. Therefore, the lower BFI and larger directional spread are observed in th south from the typhoon center. Although nonlinear effects can be neglected in the third quadrant, the bimodal situation is another issue to be discussed in detail.

The sensitivity of the nonlinear transfer function of the source term is examined to the freak wave parameter. The two different nonlinear transfer functions, DIA and MDIA, are selected and it is integrated the same typhoon condition. Fig.6 shows the time series of $H_{\max } / H_{s}$ by DIA and MDIA in the case of $r=50$ and $V=50 \mathrm{~km} / \mathrm{s}$. Overall, the differences between DIA and MDIA are small. As shown in Fig.3, the south of typhoon eye becomes bimodal wave field, therefore, strong nonlinear interactions are expected. The exact nonlinear transfer will be examined in future.

\section{CONCLUSION}

The parametric survey has carried out to obtain the general behavior of freak wave parameters, Benjamin-Feir Index (BFI), kurtosis and directional spread in the typhoon condition. The occurrence freak wave is increased as BFI increased and as directional dispersion effect becomes significant, the kurtosis is dramatically decreased in previous study.

A series of model typhoon experiments was conducted using the spectral wave model with the super gradient wind changing typhoon velocity $V$ and its radius $r$. Summarizing the numerical results, the wave field in the fourth quadrant of typhoon (southeast from typhoon eye in the Northern hemisphere) has larger BFI and narrower directional spread. The nonlinear enhancement of maximum wave in the fourth quadrant is 3-5\% larger than surround area, although it is almost linear in the third quadrant. Therefore, the wave field in the fourth quadrant of typhoon (southeast from typhoon eye) is much dangerous for freak wave occurrence than the other quadrants.

The careful validation of the numerical experiments by the observed data will be required near future. In addition, the wave growth of the typhoon condition was discussed in here and that condition is relatively young wave age. Therefore, more longer wave age conditions such as extra-tropical storm or winter seasonal storm at high latitude should be discussed near future. 


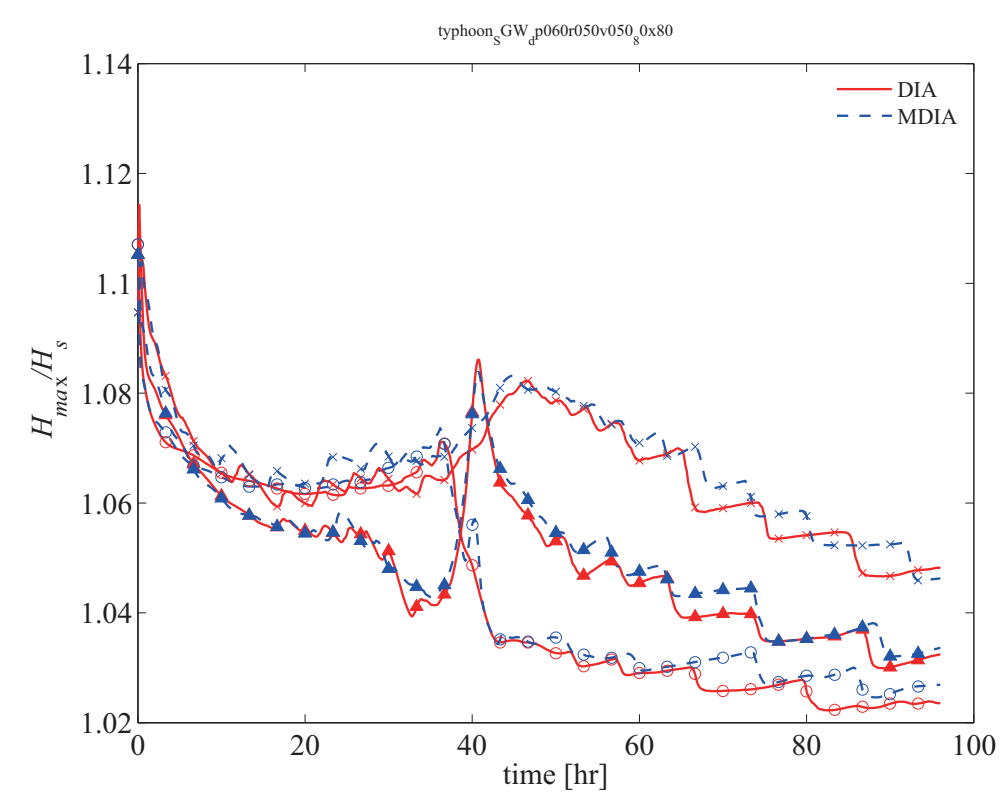

Figure 6. Time series of $H_{\max } / H_{s}$ by different nonlinear source terms (DIA, center and MDIA; $\Delta p=60 \mathbf{h P a}, r=50 \mathbf{k m}, V=50 \mathrm{~km} / \mathbf{s}$ )

\section{ACKNOWLEDGMENTS}

This research was partially supported by the Ministry of Education, Science, Sports and Culture, Japan, through Grant-in-Aid (PI: N.M.).

\section{REFERENCES}

Booij, N., R. Ris, and L. Holthuijsen. 1999. A third-generation wave model for coastal regions. IModel description and validation, Journal of Geophysical Research, 104(C4), 7649-7666.

Dean, R.. 1990. Freak waves: a possible explanation, A. Tørum and O. Gudmestad (Eds.), Water wave kinematics, 609-612. Kluwer Academic Pub.

Dysthe, K., H. Krogstad, and P. Müller. 2008. Oceanic Rogue Waves, Annual Review of Fluid Mechanics, 40, in print.

Fujii, T. and Y. Mitsuta. 1986. Simulation of winds in typhoons by a stochastic model, J. Wind Eng, 28, 1-12.

Gramstad, O. and K. Trulsen. 2007. Influence of crest and group length on the occurrence of freak waves, Journal of Fluid Mechanics, 582, 463-472.

Guedes Soares, G., Z. Cherneva, and E. Antao. 2003. Characteristics of abnormal waves in North Sea storm sea steas, Applied Ocean Research, 25, 337-344.

Guedes Soares, G., Z. Cherneva, and E. Antao. 2004. Abnormal waves during hurricane Camille, Journal of Geophysical Research, 109, C08008.

Janssen, P. A.. 2003. Nonlinear four-wave interactions and freak waves, Journal of Physical Oceanography, 33(4), 863-884. 


\section{COASTAL ENGINEERING 2010}

Lavrenov, I.. 1998. The wave energy concentration at the Agulhas current off South Africa, Natural Hazard, 17, 117-127.

Longuet-Higgins, M.. 1963. The effect of non-linearities on statistical distirbutions in the theory of sea waves, Journal of Fluid Mechanics, 17, 459-480.

Mori, N. and P. Janssen. 2006. On kurtosis and occurrence probability of freak waves, Journal of Physical Oceanography, 36(7), 1471-1483.

Mori, N., P. Liu, and T. Yasuda. 2002. Analysis of freak wave measurements in the Sea of Japan, Ocean Engineering, 29(11), 1399-1414.

Mori, N., M. Onorato, and P. A. Janssen. 2010. On the estimation of the kurtosis in directional sea states for freak wave forecasting, Journal of Physical Oceanography,, submitted.

Mori, N., M. Onorato, P. A. Janssen, A. R. Osborne, and M. Serio. 2007. Exceedance probability for strongly nonlinear long crested waves, Journal of Geophysical Research, doi:10.1029/2006JC004024.

Onorato, M., L. Cavaleri, S. Fouques, O. Gramstad, and P. A. E. M. Janssen. 2009. Statistical properties of mechanically generated surface gravity waves: a laboratory experiment in a $3 \mathrm{~d}$ wave basin, Journal of Fluid Mechanics, in press.

Onorato, M., A. Osborne, and M. Serio. 2005. On deviations from Guassian statistics for surface gravity waves, Proceedings Rogue Waves, Hawaiian Winter Workshop, 79-83.

Onorato, M., A. Osborne, M. Serio, and S. Bertone. 2001. Freak waves in random oceanic sea states, Physical Review Letters, 86(25), 5831-5834.

Onorato, M., T. Waseda, A. Toffoli, L. Cavaleri, O. Gramstad, P. Janssen, T. Kinoshita, J. Monbaliu, N. Mori, A. Osborne, et al.. 2009. Statistical properties of directional ocean waves: The role of the modulational instability in the formation of extreme events, Physical Review Letters, 102(11), 114502.

Osborne, A., M. Onorato, M. Serio, and S. Bertone. 2000. The nonlinear dynamics of rogue waves and holes in deep water gravity wave trains, Physical Letter A, 275(5-6), 386-393.

Petrova, P. and C. Guedes Soares. 2008. Maximum wave crest and height statistics of irregular and abnormal waves in an offshore basin, Applied Ocean Research, 30(2), 144-152.

Stansell, P., J.Wolfram, and S. Zachary. 2003. Horizontal asymmetry and steepness distributions for wind-driven ocean waves, Applied Ocean Research, 25, 137-155.

Trulsen, K. and K. Dysthe. 1997. Freak waves - A three-dimensional wave simulation, Proceedings of the 21st Symposium on Naval Hydrodynamics, 550-558. (National Academy Press.

Waseda, T.. 2006. Impact of directionality on the extreme wave occurrence in a discrete random wave system, The 9th Wave Workshop, 10p.

Yasuda, T. and N. Mori. 1994. High order nonlinear effects on deep-water random wave trains, International Symposium: Waves-Physical and Numerical Modelling, Volume 2, Vancouver, 823332 .

Yasuda, T. and N. Mori. 1997. Occurrence properties of giant freak waves the sea area around Japan, 123(4), 209-213. 


\section{COASTAL ENGINEERING 2010}

Yasuda, T., N. Mori, and K. Ito. 1992. Freak waves in a unidirectional wave train and their kinematics, Proc. of the 23th Int. Conf. on Coastal Eng., Volume 1, Venice, ASCE, 751-764.

Yasuda, T., N. Mori, and S. Nakayama. 1997. Characteristics of giant freak waves observed in the Sea of Japan, Waves97, Virginia, VA, 482-495.

Young, I.. 2006. Directional spectra of hurricane wind waves, Journal of Geophysical Research, 111(C8), C08020. 